\title{
On the macroion virial contribution to the osmotic pressure in charge-stabilized colloidal suspensions
}

\author{
E. Trizac \\ NSF Center for Theoretical Biological Physics, UCSD, La Jolla, CA 92093-0374 USA and \\ LPTMS, Univ. Paris-Sud, UMR 8626, Orsay F-91405 and CNRS, Orsay F-91405 \\ Luc Belloni \\ Laboratoire Interdisciplinaire sur l'Organisation Nanométrique et Supramoléculaire CEA/SACLAY, \\ Service de Chimie Moléculaire, 91191 Gif sur Yvette Cedex, France \\ J. Dobnikar \\ Institut für Chemie, Karl-Franzens-Universität, Heinrichstrasse 28, 8010 Graz, Austria and \\ Jozef Stefan Institute, Jamova 39, 1000 Ljubljana, Slovenia \\ H.H. von Grünberg \\ Institut für Chemie, Karl-Franzens-Universität, Heinrichstrasse 28, 8010 Graz, Austria \\ R. Castañeda-Priego \\ Instituto de Física, Universidad de Guanajuato, 37150 Leon, Mexico
}

(Dated: October 4, 2018)

\begin{abstract}
Our interest goes to the different virial contributions to the equation of state of charged colloidal suspensions. Neglect of surface effects in the computation of the colloidal virial term leads to spurious and paradoxical results. This pitfall is one of the several facets of the danger of a naive implementation of the so called One Component Model, where the micro-ionic degrees of freedom are integrated out to only keep in the description the mesoscopic (colloidal) degrees of freedom. On the other hand, due incorporation of wall induced forces dissolves the paradox brought forth in the naive approach, provides a consistent description, and confirms that for salt-free systems, the colloidal contribution to the pressure is dominated by the micro-ionic one. Much emphasis is put on the no salt case but the situation with added electrolyte is also discussed.
\end{abstract}

\section{INTRODUCTION}

In a complex mixture where several species with widely different characteristic time and space scales coexist, it is common practice to resort to a coarse grained description integrating from the partition function all degrees of freedom that do not belong to the main (larger) constituent [1, 2, 3, 4]. This leads to a state dependent effective Hamiltonian for the main constituent, thereby allowing a One Component Model (OCM) description. The motivation for such a procedure is not only to facilitate contact with experiments, where most of the time the small constituents cannot be probed directly, but also to simplify the theoretical treatment. Indeed, one can then use the well developed statistical mechanics tools from the theory of simple liquids to study the OCM. This transposition from simple to complex fluids is however paved with practical difficulties, see e.g. [5, 6]. It is the purpose of the present paper to discuss one such pitfall arising in the context of charged colloidal suspensions.

The system we will consider is made up of $N_{c}$ charged spherical hard particles (colloids) immersed in a solvent with dielectric constant $\varepsilon$, which fills a box with volume $V$ limited by a neutral hard wall. The colloid's interior is assumed to have the same dielectric constant as the solvent. Each colloid bears a charge $Z_{c} e$ where $e$ is the elementary charge and $Z_{c} \gg 1$. The medium outside the container is a structureless dielectric continuum with dielectric constant $\varepsilon^{\prime}$. To ensure electroneutrality, the solution contains $N_{c} Z_{c}$ microscopic counterions, assumed monovalent. Additional microions may also be present due to the dissociation of an added salt and the total number of microions is denoted $N_{\text {micro }}$. The particles interact through Coulomb forces and hard sphere exclusion, although in the subsequent analysis, the hard-core interaction will turn out to be irrelevant.

The paper is organized as follows. In the situation where $\varepsilon=\varepsilon^{\prime}$, we consider in section II the different virial contributions to the equation of state. In the salt-free case, we argue that the colloidal contribution $P_{\mathrm{ocm}}$ has to be negligible compared to the microionic one $\left(P_{\text {micro }}\right)$. We then show that a naive implementation of the OCM picture

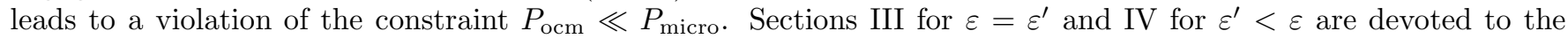
resolution of this apparent paradox. It will be shown that in a closed cell, the surface contribution to the colloidal virial $P_{\mathrm{ocm}}$ is comparable to the bulk term, while only the latter is considered in the naive picture. Hence its failure, resulting from a gross overestimation of $P_{\mathrm{ocm}}$. As a consequence, the knowledge of a good effective potential in the 
bulk is insufficient when it comes to directly computing the colloidal virial in a closed box. Concluding remarks are drawn in section $\nabla$, where we discuss in particular how the effective potential can be used -indirectly but from a standard procedure- to compute the total pressure of the system. While most of the analysis holds without salt, the situation of an added electrolyte is also briefly addressed.

\section{EQUATION OF STATE, EFFECTIVE INTERACTIONS AND ONE COMPONENT MODEL VIRIAL}

\section{A. The equation of state}

We start by the simplest situation where $\varepsilon=\varepsilon^{\prime}$ and consider all charged species in the solution. The virial theorem allows to write the total osmotic pressure $P$ (with respect to pure solvent) in the form

$$
\beta P V=N_{c}+N_{\text {micro }}+\frac{\beta}{3}\left\langle\sum_{i \in c o l+m i c r o} \boldsymbol{r}_{i} \cdot \boldsymbol{F}_{i}^{\text {int }}\right\rangle,
$$

where $\beta=1 /(k T)$ is the inverse temperature and the summation runs over colloids and microscopic ions, therefore involving $N_{c}+N_{\text {micro }}$ terms. In Eq. (1) the angular brackets denote a statistical average (that coincides with time average) and $\boldsymbol{F}_{i}^{\text {int }}$ is the (internal) force exerted on particle $i$ at position $\boldsymbol{r}_{i}$, due to hard core and Coulombic interactions in the solution. Adding the force exerted by the wall to $\boldsymbol{F}_{i}^{\text {int }}$ would therefore provide the total force $\boldsymbol{F}_{i}^{\text {tot }}$ felt by particle $i$. We note here that it is possible to express the pressure in Eq. (1) as a surface integral over the wall of the total (colloid + micro-ions) concentration. Applying the virial theorem to the microions only, we have

$$
N_{\text {micro }} k T+\frac{1}{3}\left\langle\sum_{i \in \text { micro }} \boldsymbol{r}_{i} \cdot \boldsymbol{F}_{i}^{\text {tot }}\right\rangle=0=N_{\text {micro }} k T+\frac{1}{3}\left\langle\sum_{i \in \text { micro }} \boldsymbol{r}_{i} \cdot \boldsymbol{F}_{i}^{\text {int }}\right\rangle-\frac{k T}{3}\left\langle\oint_{\text {box }} \rho_{\text {micro }}(\boldsymbol{r}) \boldsymbol{r} \cdot d^{2} \boldsymbol{S}\right\rangle,
$$

where the surface integral with normal oriented outward runs over the box confining the system. Inserting the latter equality into (10), we obtain

$$
P=\rho_{c} k T+P_{\mathrm{ocm}}+P_{\text {micro }} \quad \text { with } \quad P_{\mathrm{ocm}}=\frac{1}{3 V}\left\langle\sum_{i \in c o l} \boldsymbol{r}_{i} \cdot \boldsymbol{F}_{i}^{\text {int }}\right\rangle ; \quad P_{\text {micro }}=\frac{k T}{3 V}\left\langle\oint_{\text {box }} \rho_{\text {micro }}(\boldsymbol{r}) \boldsymbol{r} \cdot d^{2} \boldsymbol{S}\right\rangle,
$$

where $\rho_{c}=N_{c} / V$ and $\rho_{\text {micro }}(\boldsymbol{r})$ denotes the total microion density at point $\boldsymbol{r}$. Within mean-field approximation, this equation may be found in [1]. The first term on the right hand side of Eq. (3) is the colloid ideal gas term which can be safely neglected in practice for the parameter range of interest here (see below). The second term -of central interest here- is the colloid-colloid virial contribution and is indexed by the subscript OCM since it would be the only term considered (apart from the ideal gas one) in the OCM approach, restricted to the mesoscopic degrees of freedom $\left\{\boldsymbol{r}_{i}\right\}_{1 \leq i \leq N_{c}}$. Indeed, the statistical average $\langle\ldots\rangle$ may be performed in two steps :

$$
P_{\mathrm{ocm}}=\frac{1}{3 V}\left\langle\sum_{i=1}^{N_{c}} \boldsymbol{r}_{i} \cdot \boldsymbol{F}_{i}^{\mathrm{int}}\right\rangle_{\text {col+micro }}=\frac{1}{3 V}\left\langle\sum_{i=1}^{N_{c}} \boldsymbol{r}_{i} \cdot\left\langle\boldsymbol{F}_{i}^{\mathrm{int}}\right\rangle_{m i c r o}\right\rangle_{c o l}=\frac{1}{3 V}\left\langle\sum_{i=1}^{N_{c}} \boldsymbol{r}_{i} \cdot \boldsymbol{F}_{i}^{\mathrm{eff}}\right\rangle_{c o l},
$$

where we have introduced the microion averaged effective force $\boldsymbol{F}_{i}^{\text {eff }}$ exerted on colloid $i$ for a given colloid configuration.

The third term in (3), $P_{\text {micro }}$, accounts for the direct coupling between colloids and microions. In principle, this third term is to be averaged over the colloidal degrees of freedom. However, even at simplified or mean-field level, a full $N_{c}$-colloid simulation is computationally demanding [7, 8, ,9], and further simplifications are helpful. Of particular interest are two such simplifications, both belonging to the Poisson-Boltzmann family, that reduce the initial $N_{c}$-body problem onto a $N_{c}=1$ body situation. The first one is the common cell model approach originating from a solid state point of view where the Wigner-Seitz cell around a colloid is constructed and then "sphericalized" for the sake of simplicity. The Poisson-Boltzmann equation is solved within this cell, and from the microionic density profile one can then estimate $P_{\text {micro }}$. The second model is the renormalized jellium model [10] where a liquid state point of view is adopted: the colloid-colloid pair distribution function $g_{c c}(r)$ is considered structureless so that other colloids around a tagged macroion behave as a continuous background. The charge of this background is a priori unknown, and enforced to coincide with the effective charge. This self-consistency requirement leads to a unique and well defined effective charge [10]. It has been shown that for salt-free suspensions, these two models - cell and jellium - both lead to a pressure $P_{\text {micro }}$ that is in excellent agreement with existing experimental data [11] and primitive model simulations 
for $P$ [12, 13], see e.g. [10, 14, 15]. We note that $P_{\text {micro }}$ may be coined a "volume" term [2, 17, 18], since - at least, within the cell model and jellium approaches - it does not depend on the colloidal degrees of freedom but only on the mean colloidal density. The good agreement one obtains with the exact pressure $P$ for both models implies that for salt-free systems $P \simeq P_{\text {micro }}$. This is corroborated by a recent study of finite stiff-chain polyelectrolytes [19]. From Eq. (3) where the ideal gas contribution $\left(\rho_{c} k T\right)$ is neglected, this may be transposed into the following requirement:

$$
P_{\text {ocm }} \ll P_{\text {micro }} \text {. }
$$

A similar conclusion was reached in Ref. [20].

\section{B. Effective interactions}

Both Poisson-Boltzmann cell and jellium approaches are not only useful to estimate the pressure, but also to derive effective parameters for solvent + microions averaged colloid/colloid interactions. By construction, the effective potential is that which leads to the correct colloid-colloid pair structure encoded in the potential of mean force $g_{c c}$, assuming pair-wise colloid-colloid interactions within the OCM model (see e.g. [1]). Although the effective potential has a clear-cut definition, there is no rigorous operational route to construct this object. In general, when microionic correlations do not invalidate the mean-field picture [13], a good approximation is to write the effective potential as a sum of pair-wise Yukawa terms of the form

$$
\beta v_{\text {eff }}(r)=Z_{\text {eff }}^{2} \lambda_{B}\left(\frac{\exp \left(\kappa_{\text {eff }} a\right)}{1+\kappa_{\text {eff }} a}\right)^{2} \frac{\exp \left(-\kappa_{\text {eff }} r\right)}{r}
$$

with $a$ the colloid radius, $\lambda_{B}=\beta e^{2} / \varepsilon$ the Bjerrum length, and $Z_{\text {eff }}$ and $\kappa_{\text {eff }}$ the effective charge and inverse screening length computed within the cell or jellium model [10, 21, 22, 23]. Such a "DLVO"-like expression [1, 2, 4] would accurately reproduce the large distance interaction of two colloids in a salt sea [1, 2, 4]. Its relevance in the no-salt case will not be discussed. As will become clear below, we are interested here in orders of magnitude, that should not depend on the precise form of (6).

\section{An apparent paradox}

Within the jellium model, the salt-free equation of state takes a particularly simple form

$$
\beta P_{\text {micro }}=Z_{\text {eff }} \rho_{c} .
$$

Within the cell model, this expression is not exact but approximately correct. For a highly charged macroion, one has $Z_{\text {eff }} \gg 1$ which allows to neglect the ideal gas term in (3). In spite of its simplicity, the expression $\beta P_{\text {micro }}=\rho_{c} Z_{\text {eff }}$ hides a complex density dependence through $Z_{\text {eff }}$ and is in excellent agreement with the exact pressure $P$ found experimentally or in primitive model simulations, as emphasized above. In addition, the effective screening length reads [10]

$$
\kappa_{\text {eff }}^{2}=4 \pi \lambda_{B} \rho_{c} Z_{\text {eff }}
$$

The constraint embodied in Eq. (5) may therefore be rewritten

$$
\beta P_{\text {ocm }} \ll Z_{\text {eff }} \rho_{c} .
$$

Alternatively, in the low electrostatic coupling regime (where $Z_{\text {eff }}$ coincides with $Z_{c}$ ), one should recover the ideal gas pressure $\beta P \simeq \rho_{c}\left(1+Z_{\text {eff }}\right)$. Given that in this limit, $\beta P_{\text {micro }} \simeq \rho_{c} Z_{\text {eff }}$, we recover the requirement (9), that will be an important benchmark for the following analysis. We now turn to the formulation of the apparent paradox.

In the bulk of the suspension, the effective potential (6) provides the effective force acting on a colloid $i$

$$
\boldsymbol{F}_{i}^{\mathrm{eff}}=\sum_{j=1}^{N_{c}} \boldsymbol{F}_{i j}^{\mathrm{eff}}=-\left.\sum_{j=1}^{N_{c}} \boldsymbol{\nabla}_{\boldsymbol{r}} v_{\mathrm{eff}}(r)\right|_{\boldsymbol{r}=\boldsymbol{r}_{i}-\boldsymbol{r}_{j}} .
$$

Considering naively that $P_{\mathrm{ocm}}$ appearing in (3) and (4) is dominated in a very large system by its bulk behaviour, we insert (10) into (4) to approximate $P_{\mathrm{ocm}}$ by $P_{\mathrm{ocm}}^{*}$ with

$$
P_{\mathrm{ocm}}^{*}=\frac{1}{3 V}\left\langle\sum_{i, j=1}^{N_{c}} \boldsymbol{r}_{i} \cdot \boldsymbol{F}_{i j}^{\mathrm{eff}}\right\rangle_{c o l}=\frac{1}{6 V}\left\langle\sum_{i, j=1}^{N_{c}} \boldsymbol{r}_{i j} \cdot \boldsymbol{F}_{i j}^{\mathrm{eff}}\right\rangle_{c o l}
$$


where $\boldsymbol{r}_{i j}=\boldsymbol{r}_{i}-\boldsymbol{r}_{j}$. We will subsequently omit the subscript "col" indicating the degrees of freedom involved in the average. Introducing the colloid-colloid pair correlation function $g_{c c}(r)$, we can write

$$
\begin{aligned}
\beta P_{\mathrm{ocm}}^{*} & =-\frac{\rho_{c}^{2}}{6} \int_{r=2 a}^{\infty} g_{c c}(r) \frac{d \beta v_{\mathrm{eff}}(r)}{d r} r d^{3} \boldsymbol{r} \\
& =\frac{2 \pi \rho_{c}^{2} Z_{\mathrm{eff}}^{2} \lambda_{B}}{\kappa_{\mathrm{eff}}^{2}}\left\{1+\frac{\left(\kappa_{\mathrm{eff}} a\right)^{2}}{3\left(1+\kappa_{\mathrm{eff}} a\right)^{2}}\right\}+\frac{\rho_{c}^{2}}{6} \int_{r=2 a}^{\infty}\left[g_{c c}(r)-1\right]\left(1+\kappa_{\mathrm{eff}} r\right) \beta v_{\mathrm{eff}}(r) d^{3} \boldsymbol{r} .
\end{aligned}
$$

To estimate the above quantity, it is sufficient to keep the dominant term only, which is the first one on the rhs, arising from the long-range behavior of the pair correlation function $\left(g_{c c} \rightarrow 1\right.$ at large distances). In this term, the curly brackets may be safely approximated by 1 since at low densities, $\kappa_{\text {eff }} a \ll 1$. Remembering Eq. (8), we obtain

$$
\begin{aligned}
P_{\mathrm{ocm}}^{*} & \simeq \frac{2 \pi \rho_{c}^{2} Z_{\mathrm{eff}}^{2} \lambda_{B}}{\kappa_{\mathrm{eff}}^{2}} \\
& \simeq \frac{1}{2} Z_{\mathrm{eff}} \rho_{c},
\end{aligned}
$$

The factor $1 / 2$ which appears is classical (see e.g. [1]). The important point here is that estimation (15) by far violates the constraint (9). A similar conclusion would be reached including the first correction in $Z_{\text {eff }}^{2} \exp \left(-\kappa_{\text {eff }} r\right) / r$ to the long distance behaviour $g=1$ when computing the integral on the rhs of (13): this yields $P_{\text {ocm }}^{*} \simeq Z_{\text {eff }} \rho_{c} / 2\left[1+\mathcal{O}\left(\kappa_{\text {eff }} \lambda_{B}\right)\right]$ with $\kappa_{\text {eff }} \lambda_{B} \ll 1$ in the dilute limit. The paradox here is that the very same approach that provides a contribution $P_{\text {micro }}$ very close to the total pressure, gives an effective potential that apparently spoils the previous agreement, by grossly overestimating the colloidal virial contribution to the pressure. We will see that this feature is not ascribable to a failure of the functional form of Eq. (6), which provides a decent approximation for the quantity $P_{\mathrm{ocm}}^{*}$.

\section{How can the paradox be resolved?}

The root of the paradox reported above is that approximating $P_{\mathrm{ocm}}$ by $P_{\mathrm{ocm}}^{*}$ is incorrect: while $P_{\mathrm{ocm}}^{*}$ provides a reasonable estimate for the bulk contribution to $P_{\mathrm{ocm}}$, surface effects make that in the vicinity of the wall, the effective force felt by a colloid differs from (10). These surface induced terms play a key role here and contribute a large amount to the colloidal virial $P_{\mathrm{ocm}}$, no matter how large the system is. It turns out that bulk and surface induced contributions almost cancel each other, so that the resulting expression for $P_{\mathrm{ocm}}$ is much smaller than $P_{\mathrm{ocm}}^{*}$ and therefore fulfills the requirement (9). Our goal in the remainder is to illustrate this cancellation explicitly, from a correct description of confinement effects. To this aim, it is judicious to simplify the problem by considering the limit of point colloids $(a=0)$, and by identifying the effective charge with the bare one $Z_{c}$. Considering charge renormalization effects is here immaterial and focussing on dilute systems where $\kappa a$ is small, finite $a$ effects do not affect our main conclusions. In the bulk of the suspension, the effective potential therefore takes a simple Yukawa form

$$
\beta v_{\text {eff }}(r)=Z_{c}^{2} \lambda_{B} \frac{\exp (-\kappa r)}{r}
$$

with $\kappa^{2}=4 \pi \lambda_{B} Z_{c} \rho_{c}$.

At this point, a comparison with simple electrolytes seems appropriate, for the aforementioned cancellation is already present. For our discussion, we may consider that the role of the colloids is played by the cations, and that the anions constitute the remaining "microions". The pressure has to be close to [1, 4]

$$
\beta P_{\text {electrolyte }} \simeq \rho_{\text {anion }}+\rho_{\text {cation }}-\frac{\kappa^{3}}{24 \pi},
$$

with equal mean densities $\rho_{\text {anion }}=\rho_{\text {cation. }}$ From the contact theorem, we deduce the densities at the wall

$$
\rho_{\text {anion }}(\text { wall })=\rho_{\text {cation }}(\text { wall }) \simeq \rho_{\text {anion }}-\frac{\kappa^{3}}{48 \pi} .
$$

Rewriting (3) in the form

$$
\beta P_{\text {electrolyte }}=\rho_{\text {cations }}+\frac{\beta}{3 V}\left\langle\sum_{i \in \text { cation }} \boldsymbol{r}_{i} \cdot \boldsymbol{F}_{i}^{\text {int }}\right\rangle+\rho_{\text {anion }}(\text { wall })
$$


we obtain from (17) and (18)

$$
\frac{\beta}{3 V}\left\langle\sum_{i \in \text { cation }} \boldsymbol{r}_{i} \cdot \boldsymbol{F}_{i}^{\mathrm{int}}\right\rangle \simeq-\frac{\kappa^{3}}{48 \pi}
$$

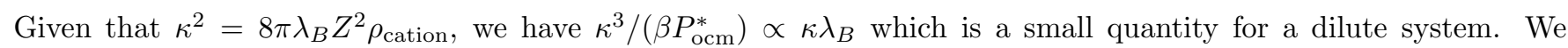
explicitly see here that the "colloidal" virial [lhs of (20) up to a factor $\beta$ ] is by far smaller than the estimation $P_{\text {ocm }}^{*}$

\section{WALL MEDIATED FORCES WITHOUT DIELECTRIC DISCONTINUITY}

In the vicinity of the wall, the colloids do not see a spherically symmetric environment. As a consequence,

1. the usual $\exp (-\kappa r) / r$ pair interaction is modified.

2. the mean force acting on a colloid does not vanish. This is a one body, wall induced effect, mediated by the microions. It is therefore an internal force, that should be taken into account in (4). It should not be confused with the external (and short range) direct colloid-wall interaction.

Evaluating the rhs of (4) therefore requires a careful computation of both types of microion averaged colloidal forces. To this end, we need the solution $\phi_{z}\left(\rho, z^{\prime}\right)$ of Debye-Hückel equation $\nabla^{2} \phi_{z}=\kappa^{2} \theta\left(z^{\prime}\right) \phi_{z}$ in the case where a test charge is located in the solution a distance $z$ from an infinite neutral wall. We have introduced the Heaviside function $\theta$ and cylindrical coordinates $\left(\rho, z^{\prime}\right)$ such that the test particle is located at $(0, z)$ with $z>0$. The planar geometry approximation for the wall is sufficient provided the cell size or radius of curvature is much larger than Debye length $1 / \kappa$. We start by the situation of equal dielectric constants inside and outside the solution $\left(\varepsilon=\varepsilon^{\prime}\right)$. The electrostatic potential may be written in the form of a Hankel (two dimensional Fourier) transform [26] where $q$ and $\rho$ are conjugate quantities [24, 25]

$$
\phi_{z}\left(\rho, z^{\prime}\right)=Z_{c} \lambda_{B} \int_{0}^{\infty}\left(\frac{k-q}{k+q} e^{-k\left(z+z^{\prime}\right)}+e^{-k\left|z-z^{\prime}\right|}\right) \frac{1}{k} J_{0}(q \rho) q d q \quad ; \quad k \equiv \sqrt{\kappa^{2}+q^{2}}
$$

The second term in the integrand $\left(e^{-\kappa\left|z-z^{\prime}\right|}\right)$ gives exactly $Z_{c} \lambda_{B} \exp (-\kappa r) / r$ where $r=\left[\rho^{2}+\left(z-z^{\prime}\right)^{2}\right]^{1 / 2}$ is the distance to the source. This is the standard Debye-Hückel potential which dominates in the bulk. The remaining term, which vanishes at large distances $\left(\kappa z\right.$ or $\left.\kappa z^{\prime} \gg 1\right)$ is due to the presence of the interface.

\section{A. One colloid ion average force}

The force felt by a colloid located a distance $z$ from the planar interface follows from (21), considering the electrostatic potential $\widetilde{\phi}_{z}=\phi_{z}-Z_{c}^{2} \lambda_{B} e^{-\kappa r} / r$ where the self term has been subtracted:

$$
\beta \boldsymbol{F}_{c-w a l l}=\left.\widehat{\mathbf{n}} Z_{c} \frac{\partial}{\partial z^{\prime}} \beta \widetilde{\phi}_{z}\left(0, z^{\prime}\right)\right|_{z, z^{\prime}=z}=Z_{c}^{2} \lambda_{B} \int_{0}^{\infty} \frac{k-q}{k+q} e^{-2 k z} q d q(-\widehat{\mathbf{n}}) .
$$

In this equation, $\widehat{\mathbf{n}}$ denotes the unit vector perpendicular to the interface pointing outside the solution. We coin the force (22) "colloid-wall" and for notational convenience, we henceforth omit the superscripts "int" and "eff". This force repels the colloid from the wall $\left[k=\left(\kappa^{2}+q^{2}\right)^{1 / 2}>q\right]$, as a result of microions imbalance between the half of the colloid exposed to the wall, and the other hemisphere. Inserting (22) into (4) we have

$$
\frac{1}{3 V}\left\langle\sum_{i=1}^{N_{c}} \boldsymbol{r}_{i} \cdot \boldsymbol{F}_{i-\text { wall }}\right\rangle=\frac{1}{3 V} \int_{\text {wall }} d^{2} S \int_{0}^{\infty} \rho_{c}(z) \boldsymbol{r} \cdot \boldsymbol{F}_{i-\text { wall }}(z) d z .
$$

To leading order, the above integral may be computed assuming a uniform density of colloids $\rho_{c}(z)=\rho_{c}$. In (23), $\boldsymbol{r}$ denotes the absolute position with $\boldsymbol{r}=\boldsymbol{s}-z \widehat{\mathbf{n}}$ ( $\boldsymbol{s}$ is therefore the orthogonal projection of $\boldsymbol{r}$ onto the wall). We 
neglect the term in $-z \widehat{\mathbf{n}}$ (that would contribute proportionally to the surface of the system), so that

$$
\begin{aligned}
\frac{1}{3 V}\left\langle\sum_{i=1}^{N_{c}} \boldsymbol{r}_{i} \cdot \beta \boldsymbol{F}_{i-\text { wall }}\right\rangle & \simeq \frac{\beta \rho_{c}}{3 V} \int_{\text {wall }} d^{2} S \int_{0}^{\infty} \boldsymbol{s} \cdot \boldsymbol{F}_{i-\text { wall }}(z) d z \\
& \simeq-\frac{\rho_{c}}{3 V} Z_{c}^{2} \lambda_{B}\left(\int_{\text {wall }} \boldsymbol{s} \cdot \widehat{\mathbf{n}} d^{2} S\right) \int_{0}^{\infty} d z \int_{0}^{\infty} \frac{\sqrt{\kappa^{2}+q^{2}}-q}{\sqrt{\kappa^{2}+q^{2}}+q} e^{-2 z \sqrt{\kappa^{2}+q^{2}}} q d q \\
& \simeq-\frac{1}{6} \rho_{c} Z_{c}^{2} \kappa \lambda_{B} \\
& \simeq-\frac{\kappa^{3}}{24 \pi} .
\end{aligned}
$$

Incidentally, this is exactly the Debye-Hückel form for the excess pressure of an electrolyte [see Eq. (17)]. For dilute systems, this quantity is small compared to $\rho_{c} Z_{c}$, as emphasized earlier. The constraint (9) is therefore fulfilled.

\section{B. Colloid-colloid interactions}

Within the simple Debye-Hückel treatment, the potential of interaction between two colloids near the wall (one at $z$, the other at $z^{\prime}$, with a lateral distance $\rho$ between them) is $Z_{c} \phi_{z}\left(\rho, z^{\prime}\right)=Z_{c} \phi_{z^{\prime}}(\rho, z)$. To calculate the force felt by the colloid at $z$ due to all neighbors, we assume again a uniform distribution of neighbors:

$$
\begin{aligned}
\beta \boldsymbol{F}_{\text {col-col }}(z) & =\left.\widehat{\mathbf{n}} \rho_{c} \int_{z^{\prime}=0}^{\infty} d z^{\prime} \int_{0}^{\infty} 2 \pi \rho d \rho \frac{\partial Z_{c} \beta \phi_{z}\left(\rho, z^{\prime}\right)}{\partial z}\right|_{z^{\prime}} \\
& =-\widehat{\mathbf{n}} \rho_{c} Z_{c}^{2} \lambda_{B} \int_{0}^{\infty} d q \int_{0}^{\infty} 2 \pi \rho d \rho\left(\frac{\sqrt{\kappa^{2}+q^{2}}-q}{\sqrt{\kappa^{2}+q^{2}}+q}-1\right) e^{-z \sqrt{\kappa^{2}+q^{2}}} \frac{1}{\sqrt{\kappa^{2}+q^{2}}} J_{0}(q \rho) q d q
\end{aligned}
$$

The component of the force parallel to the wall vanishes upon averaging.

Inserting this force into (4) and proceeding along similar lines as in Eqs. (24) sq, we have

$$
\frac{\beta}{3 V}\left\langle\sum_{i=1}^{N_{c}} \boldsymbol{r}_{i} \cdot \boldsymbol{F}_{i-\text { coll }}\right\rangle \simeq \rho_{c}^{2} Z_{c}^{2} \lambda_{B} \int_{0}^{\infty} 2 \pi \rho d \rho \int_{0}^{\infty}\left(-\frac{\sqrt{\kappa^{2}+q^{2}}-q}{\sqrt{\kappa^{2}+q^{2}}+q}+1\right) \frac{1}{\kappa^{2}+q^{2}} J_{0}(q \rho) q d q .
$$

Both expressions (29) and (30) are of the form of a Hankel transform at the origin $q=0$ of the inverse Hankel transform of a function $A(q)$, with $A=(\ldots-1) e^{-k z} / k$ in (29) and $A=(-\ldots+1) / k^{2}$ in (30). This is nothing but $A(0)$ [26], which vanishes in both cases. Therefore, with the approximations proposed, the force in (29) and the virial term in (30) vanish. To be more specific, we compute explicitly the integrals in (30):

$$
\frac{\beta}{3 V}\left\langle\sum_{i=1}^{N_{c}} \boldsymbol{r}_{i} \cdot \boldsymbol{F}_{i-\text { coll }}\right\rangle \simeq \frac{1}{2} \rho_{c} Z_{c}(-1+1)
$$

The term in +1 in the parenthesis arises from the term in +1 in Eq. (30), which gives the usual "bulk" $e^{-\kappa r} / r$ pair interaction, as already mentioned. The associated virial is $\beta P_{\mathrm{ocm}}^{*}=Z_{c} \rho_{c} / 2$, as obtained in (15). The present calculation shows that this term is canceled by an opposite wall induced contribution. If the simplifying assumption $g_{c c}=1$ is relaxed, the resulting expression for (31) no longer vanishes but remains negligible with respect to $\rho_{c} Z_{c}$. On the other hand, relaxing the assumption of a uniform profile $\rho_{c}(z)$ leaves the result unaffected, as will be seen in section IV.

We conclude here that summing the two contributions from Eqs. (27) and (31) provides a value for $P_{\mathrm{ocm}}$ that is compatible with the constraint (9).

\section{ANALYSIS IN PRESENCE OF A DIELECTRIC DISCONTINUITY}

In this section, we extend the previous analysis to the situation where the dielectric constants are not matched: $\eta=\varepsilon^{\prime} / \varepsilon \neq 1$. The relevant parameter range corresponds to $\eta<1$ e.g for water droplets in air in a spray-drying experiments. The first important difference with the $\eta=1$ case is that the equation of state (3) takes a different 
form. The pressure is indeed not solely given by the contact densities of charged species at the wall, but contains additional electric contributions (polarization or image effects). On the other hand, Eqs (11) and (2) are still formally correct provided one also includes in the "internal" forces the electric forces from the wall. The resulting equation of state reads

$$
P=\rho_{c} k T+\frac{1}{3 V}\left\langle\sum_{i \in c o l} \boldsymbol{r}_{i} \cdot \boldsymbol{F}_{i}^{\mathrm{int}}\right\rangle+\frac{k T}{3 V}\left\langle\oint_{\mathrm{box}} \rho_{\text {micro }}(\boldsymbol{r}) \boldsymbol{r} \cdot d^{2} \boldsymbol{S}\right\rangle+\frac{1}{3 V}\left\langle\oint_{\mathrm{box}} \boldsymbol{r} \cdot \boldsymbol{T}^{e l} d \boldsymbol{S}\right\rangle .
$$

Here

$$
\boldsymbol{T}^{e l}=\frac{\varepsilon}{8 \pi} E^{2} \boldsymbol{I}-\frac{\varepsilon}{4 \pi} \boldsymbol{E} \otimes \boldsymbol{E}
$$

is the Maxwell tensor, with $\boldsymbol{E}$ the local electric field and $\boldsymbol{I}$ the isotropic tensor.

The counterpart of (21) now reads:

$$
\phi_{z}\left(\rho, z^{\prime}\right)=Z_{c} \lambda_{B} \int_{0}^{\infty}\left(\frac{k-\eta q}{k+\eta q} e^{-k\left(z+z^{\prime}\right)}+e^{-k\left|z-z^{\prime}\right|}\right) \frac{1}{k} J_{0}(q \rho) q d q \quad ; \quad k \equiv \sqrt{\kappa^{2}+q^{2}} .
$$

As in the case $\eta=1$ and as long as $\eta \neq 0$, the corresponding interaction between two colloids decays as $\rho^{-3}$ at large distances parallel to the wall (see [27] for a discussion of this dipolar-like term). When $\eta=0$, the wall can be formally removed considering the electric image located symmetric to the $z=0$ plane.

The colloid-colloid and colloid-wall interactions readily follow from (34). At short distances $z \rightarrow 0$, the latter diverges like $z^{-1}(1-\eta) /(1+\eta)$ [25], which corresponds to the unscreened interaction of a particle with its own image. This divergence means that the uniform colloid density cannot be invoked when it comes to computing (23). To obtain the leading order behaviour, we can assume that the colloids are distributed with the Boltzmann weight $\rho_{c}(z)=\rho_{c} \exp \left[-\beta \phi_{c-\text { wall }}(z)\right]$, where $\boldsymbol{F}_{c-\text { wall }}=-\boldsymbol{\nabla} \phi_{c-\text { wall }}$ and the potential $\phi_{c-\text { wall }}$ deriving from (34) vanishes for $z \rightarrow \infty$. The precise knowledge of this potential is however not required since

$$
\begin{aligned}
\frac{1}{3 V}\left\langle\sum_{i=1}^{N_{c}} \boldsymbol{r}_{i} \cdot \boldsymbol{F}_{i-\text { wall }}\right\rangle & =\frac{\rho_{c}}{3 V} \int_{\text {wall }} d^{2} S \int_{0}^{\infty} \boldsymbol{r} \cdot \boldsymbol{F}_{c-\text { wall }}(z) \exp \left[-\beta \phi_{c-\text { wall }}(z)\right] d z \\
& \simeq \rho_{c} k T\left[\exp \left(-\beta \phi_{c-\text { wall }}(z)\right)\right]_{0}^{\infty} \\
& \simeq-\rho_{c} k T .
\end{aligned}
$$

This term therefore cancels the ideal gas one on the rhs of (32).

The wall induced colloid-colloid contribution to the colloidal virial may be computed along similar lines as in section IIB. An expression involving again a Hankel transform composed with its inverse is again obtained, with now a function

$$
A(q)=\left(\frac{\sqrt{\kappa^{2}+q^{2}}-\eta q}{\sqrt{\kappa^{2}+q^{2}}+\eta q}-1\right) \frac{1}{\sqrt{\kappa^{2}+q^{2}}} \int_{0}^{\infty} d z \rho_{c}(z) e^{-z \sqrt{\kappa^{2}+q^{2}}}
$$

Since $A(0)=0$, we conclude here that

$$
\left\langle\sum_{i=1}^{N_{c}} \boldsymbol{r}_{i} \cdot \boldsymbol{F}_{i-\text { coll }}\right\rangle \simeq 0
$$

so that the total colloidal virial [including colloid-colloid and colloid-wall interactions] is close to $-\rho_{c} k T$, which is a small quantity compared to the microionic contribution $Z_{c} \rho_{c} k T$. Equation (32) can finally be rewritten

$$
P \simeq \frac{k T}{3 V}\left\langle\oint_{\mathrm{box}} \rho_{\mathrm{micro}}(\boldsymbol{r}) \boldsymbol{r} \cdot d^{2} \boldsymbol{S}\right\rangle+\frac{1}{3 V}\left\langle\oint_{\mathrm{box}} \boldsymbol{r} \cdot \boldsymbol{T}^{e l} d \boldsymbol{S}\right\rangle
$$

\section{CONCLUDING REMARKS}

Before briefly discussing the situation where a salt is added, two comments are in order. 


\section{A. Closed cells versus periodic boundary conditions}

From the previous discussion, it appears that the equation of state (3) holds when the system is confined by a hard wall, and would fail if periodic boundary conditions ( $\mathrm{pbc}$ ) would be enforced. The inadequacy of $P_{\mathrm{ocm}}^{*}$ to approximate $P_{\text {ocm }}$ may then be phrased in the following way

$$
\begin{aligned}
3 V P_{\mathrm{ocm}}^{*} & \equiv \frac{1}{2}\left\langle\sum_{i, j=1}^{N_{c}} \sum_{\mathbf{n}} \boldsymbol{r}_{i j} \cdot \boldsymbol{F}_{i j}^{\mathrm{eff}}\left(\boldsymbol{r}_{i j}-\boldsymbol{R}_{\mathbf{n}}\right)\right\rangle_{p b c} \\
& \neq\left\langle\sum_{i}^{N_{c}} \boldsymbol{r}_{i} \cdot \boldsymbol{F}_{i}^{\mathrm{eff}}\right\rangle_{\text {hard walls }}
\end{aligned}
$$

where in (41), the sum involves all periodic images of the cell considered: $\mathbf{n}$ is a vector with components in $\mathbb{Z}^{3}$, which indexes the center $\mathbf{R}_{\mathbf{n}}$ of a given image of the "central" cell. The central cell has $\mathbf{R}_{\mathbf{0}}=\mathbf{0}$ and since we deal here with a short range effective potential, the sum over $\mathbf{n}$ may be truncated to retain only the 7 terms with $|\mathbf{n}| \leq 1$.

However, for any simple fluid where the forces $\boldsymbol{F}_{i}$ are given, (42) would be an equality. Indeed we have

$$
\left\langle\sum_{i} \boldsymbol{r}_{i} \cdot \boldsymbol{F}_{i}\right\rangle_{\text {hard walls }}^{\text {simple fluid }} \equiv \frac{1}{2}\left\langle\sum_{i, j} \boldsymbol{r}_{i j} \cdot \boldsymbol{F}_{i j}\right\rangle_{\text {hard walls }}^{\text {simple fluid }}
$$

where the rhs shows negligible dependence on the boundary conditions provided the system is large enough, and can then be computed with pbc provided the correct forces are considered $\left[\boldsymbol{F}_{i}=\sum_{j} \sum_{\mathbf{n}} \boldsymbol{F}_{i j}\left(\boldsymbol{r}_{i j}-\boldsymbol{R}_{\mathbf{n}}\right)\right]$. Hence

$$
\left\langle\sum_{i} \boldsymbol{r}_{i} \cdot \boldsymbol{F}_{i}\right\rangle_{\text {hard walls }}^{\text {simple fluid }}=\frac{1}{2}\left\langle\sum_{i, j} \sum_{\mathbf{n}} \boldsymbol{r}_{i j} \cdot \boldsymbol{F}_{i j}\left(\boldsymbol{r}_{i j}-\boldsymbol{R}_{\mathbf{n}}\right)\right\rangle_{p b c}^{\text {simple fluid }}
$$

The difference between equations (42) and (44) illustrates the important role of microions. We may also consider that the $\neq$ sign in (42) arises from the density dependence of the effective pair potential.

A natural question at this point is : does the knowledge of the "bulk" effective potential (6) between colloids allow to compute their virial $P_{\mathrm{ocm}}$ as it appears in (3) ? The answer is positive in a closed cell, at the OCM level, provided that due account is taken for the dielectric images of the colloids. In the following section, we address a related question, and discuss how the full pressure of the colloidal system may be recovered, assuming again that the only information at hand is that of the bulk effective colloid-colloid interaction.

\section{B. Back to the DLVO potential}

We consider here a simple liquid that interacts with a pair-wise potential given by Eq. (6), with effective parameters $Z_{\text {eff }}^{*} \gg 1$ and $\kappa_{\text {eff }}^{* 2}=4 \pi \lambda_{B} Z_{\text {eff }}^{*} \rho_{c}^{*}$ (salt-free case, for simplicity). These parameters are fixed a priori, and chosen to coincide with those relevant for a colloidal suspension at $\rho_{c}=\rho_{c}^{*}$. The potential of interaction is therefore density independent and the system, later referred to as "auxiliary", can be studied for $\rho_{c} \neq \rho_{c}^{*}$.

We consider the parameter range (essentially low density) where the excess pressure of such a system is well approximated by $P_{\mathrm{ocm}}^{*}$ in Eq. (14):

$$
\beta P_{\mathrm{ocm}}^{*} \simeq \frac{2 \pi \rho_{c}^{2} Z_{\mathrm{eff}}^{* 2} \lambda_{B}}{\kappa_{\mathrm{eff}}^{* 2}}=\frac{1}{2} \frac{\rho_{c}^{2}}{\rho_{c}^{*}} Z_{\mathrm{eff}}^{*} .
$$

Incidentally, the contact theorem indicates that the contact density in the case where the system is confined by a closed box, reads $\rho_{c}($ wall $) \simeq \rho_{c}^{2} Z_{\text {eff }}^{*} /\left(2 \rho_{c}^{*}\right)$. This quantity is much larger than the mean density $\rho_{c}$ (except when $\rho_{c}$ is extremely small, a limit of little interest here). This excess with respect to the mean density is to be contrasted with the depletion from the wall that is present in the original colloidal system containing microions: Eq. (22) for $\varepsilon=\varepsilon^{\prime}$ shows a repulsive colloid-wall behaviour, and the depletion is even stronger when $\varepsilon^{\prime}<\varepsilon$ due to like-sign images, see the discussion after Eq. (34).

The pressure of the simple liquid with DLVO interactions, close to $P_{\mathrm{ocm}}^{*}$, has a priori nothing to do with the pressure $P^{\text {original }}$ of the real colloidal system. It has also nothing to do with the colloid virial contribution entering Eq. (3). However, for $\rho_{c}=\rho_{c}^{*}$, the colloid-colloid structural information is the same for both original and auxiliary systems. 
One may then invoke Kirkwood-Buff identity [28] which states that the inverse compressibility of the original colloidal suspension coincides with the long wave-length limit of the colloid-colloid structure factor $S_{c c}(k)$ :

$$
\chi=\left(\left.\frac{\partial \beta P^{\text {original }}}{\partial \rho_{c}}\right|_{T}\right)^{-1}=S_{c c}(0) .
$$

The compressibility in our auxiliary simple liquid with fixed potential of interaction is therefore the same at $\rho_{c}=\rho_{c}^{*}$ (and only at this density)

$$
\left.\left.\frac{\partial P^{\text {original }}}{\partial \rho_{c}}\right|_{T} \stackrel{\rho_{c}=\rho_{c}^{*}}{=} \frac{\partial P_{\mathrm{ocm}}^{*}}{\partial \rho_{c}}\right|_{T, \kappa_{\mathrm{eff}}^{*}, Z_{\mathrm{eff}}^{*}}
$$

This offers a means to compute the equation of state of the original colloidal system from integrating the inverse compressibility of the auxiliary one. In this integration, due account must be taken of the density dependence of both $Z_{\text {eff }}^{*}$ and $\kappa_{\text {eff }}^{*}$. The previous integration procedure therefore requires to consider the auxiliary system for several values of $\rho_{c}$ for a given $\rho_{c}^{*}$ [to compute the derivative in the rhs of (47)], before scanning the range of interest for $\rho_{c}^{*}$. Of course, the general procedure outlined here does not depend on the specific form of the effective potential, and is equally valid when salt is added. It turns however that the DLVO potential together with the salt-free approximation (14) - which leads to (45)- provide a clear illustration of the procedure. From (45), we obtain the rhs of Eq. (47):

$$
\left.\frac{\partial \beta P_{\mathrm{ocm}}^{*}}{\partial \rho_{c}}\right|_{T, \kappa_{\mathrm{eff}}^{*}, Z_{\mathrm{eff}}^{*}} \simeq Z_{\mathrm{eff}}^{*} \text { at } \rho_{c}=\rho_{c}^{*}
$$

To compute the lhs of (47), we may come back to the jellium model which gives $\beta P^{\text {original }} \simeq Z_{\text {eff }} \rho_{c}$. In this expression, the effective charge may depend on the density, but for salt-free cases, this dependence is at most logarithmic for $\rho_{c} \rightarrow 0$ [10] and provides only a subdominant term to the compressibility, so that

$$
\left.\frac{\partial \beta P^{\text {original }}}{\partial \rho_{c}}\right|_{T} \simeq Z_{\text {eff }}
$$

Evaluating this expression at $\rho_{c}=\rho_{c}^{*}$ where $Z_{\text {eff }}=Z_{\text {eff }}^{*}$, we recover Eq. (48). This not only illustrates the identity (47) but also the consistency of the underlying DLVO potential.

\section{Situation with added salt}

When the suspension is dialyzed against a salt reservoir, most of the technical analysis carried out earlier is still valid. We consider a similar auxiliary system as in section $\mathrm{VB}$, with effective screening length such that $\kappa_{\text {eff }}^{* 2}>4 \pi \lambda_{B} Z_{\text {eff }}^{*} \rho_{c}$ due to the screening by salt ions [29]. The effective charge and screening lengths are again chosen to coincide with those of a colloidal system at a particular density $\rho_{c}^{*}$, but are otherwise density independent. Equation (47) still holds while $P_{\mathrm{ocm}}^{*}$ is given by (13). Neglecting again the integral on the rhs of (13), and inserting the resulting $P_{\mathrm{ocm}}^{*}$ in (47), we obtain:

$$
\left.\frac{\partial P^{\text {original }}}{\partial \rho_{c}}\right|_{T}=\mathcal{A} \frac{4 \pi \lambda_{B} \rho_{c} Z_{\text {eff }}^{2}}{\kappa_{\text {eff }}^{2}}
$$

where we have replaced $Z_{\text {eff }}^{*}$ by $Z_{\text {eff }}$ and $\kappa_{\text {eff }}^{*}$ by $\kappa_{\text {eff }}$ after computing the rhs of (47). Here, the prefactor $\mathcal{A}$ reads

$$
\mathcal{A}=1+\frac{\left(\kappa_{\text {eff }} a\right)^{2}}{3\left(1+\kappa_{\text {eff }} a\right)^{2}} \text {. }
$$

Is relation (50) compatible with $P_{\mathrm{ocm}} \ll P=P^{\text {original }}$ ? Neglecting $P_{\mathrm{ocm}}$ (together with $\rho_{c} k T$ ) in (3), we have $P \simeq P_{\text {micro }}$ which in the jellium model is given by $\kappa_{\text {eff }}^{2} /\left(4 \pi \lambda_{B}\right)$. With the help of [29], we arrive at

$$
\left.\frac{\partial P_{\text {micro }}}{\partial \rho_{c}}\right|_{T}=\frac{4 \pi \lambda_{B} \rho_{c} Z_{\text {eff }}^{2}}{\kappa_{\text {eff }}^{2}} .
$$

Equations (501) and (52) give the same result provided $\mathcal{A}$ is close to unity, which means $\kappa_{\text {eff }} a<1$. We conclude here that omitting the colloidal contribution to the pressure, $P_{\mathrm{ocm}}$, is inconsistent when $\kappa_{\text {eff }} a>1$. It turns out however that $\mathcal{A}$ increases very mildly with $\kappa_{\text {eff }} a$ (e.g it is close to 1.2 for $\kappa_{\text {eff }} a=4$ ). A more precise discussion would require to consider the full rhs in (13), which is beyond the scope of this paper. Finally, we note that in the salt-free case where $\kappa_{\text {eff }} a=3 \eta_{c} Z_{\text {eff }} \lambda_{B} / A$ with $\eta_{c}=4 \pi \rho_{c} a^{3} / 3$ the colloidal volume fraction and $Z_{\text {eff }} \lambda_{B} / a$ on the order of 10 for highly charged colloids, we have $\kappa_{\text {eff }} a<3$ and therefore $\mathcal{A}$ close to 1 even for packing fractions as high as $10 \%$. 


\section{Summary}

We have seen that for a salt-free colloidal suspension, the colloidal contribution $P_{\mathrm{ocm}}$ to the equation of state [as written in Eq. (3)] is a negligible quantity. This feature may easily be overlooked in a naive implementation of the One Component Model, where only $P_{\mathrm{ocm}}^{*}$, the bulk contribution to $P_{\mathrm{ocm}}$, is computed. The fact that $P_{\mathrm{ocm}}^{*}$ is of the same order of magnitude as the total pressure $P$ of the suspension, is not compatible with the requirement $P_{\text {ocm }} \ll P_{\text {micro }} \simeq P$, that has emerged as a central constraint in our analysis. We have shown that no matter how large the system is, surface effects that require the resolution of Poisson's equation in the vicinity of a confining wall, contribute a large amount to $P_{\mathrm{ocm}}$. To zeroth approximation, these surface terms cancel the bulk value $P_{\mathrm{ocm}}^{*}$, so that one finally recovers $P_{\mathrm{ocm}} \ll P$.

\section{Acknowledgments}

It is a pleasure to thank Y. Levin for fruitful discussions. R.C.P thanks PROMEP-Mexico and CONACyT (grant 46373/A-1) for financial support. J.D. acknowledges the Marie-Curie fellowship MEIF-CT-2003-501789. This work has been supported in part by the NSF PFC-sponsored Center for Theoretical Biological Physics (Grants No. PHY0216576 and PHY-0225630). E.T. acknowledges the French ANR for an ACI.

[1] L. Belloni, J. Phys.: Condens. Matter 12, R549 (2000).

[2] J.-P. Hansen and H. Löwen, Annu. Rev. Phys. Chem. 51, 209 (2000).

[3] C.N. Likos, Phys. Rep. 348, 267 (2001).

[4] Y. Levin, Rep. Prog. Phys. 65, 1577 (2002).

[5] A.A. Louis, J. Phys.: Condens. Matt. 14, 9187 (2002).

[6] C.F. Tejeiro and M. Baus, J. Chem. Phys. 118, 892 (2003).

[7] M. Fushiki, J. Chem. Phys. 97, 6700 (1992).

[8] H. Löwen, J.P. Hansen, and P.A. Madden, J. Chem. Phys. 98, 3275 (1993).

[9] J. Dobnikar, D. Haložan, M. Brumen, H. H. von Grünberg and R. Rzehak, Comput. Phys. Commun. 159, 73 (2004).

[10] E. Trizac and Y. Levin, Phys. Rev. E 69, 031403 (2004).

[11] V. Reus, L. Belloni, T. Zemb, N. Lutterbach, and H. Versmold, J. Phys. II France 7, 603 (1997).

[12] P. Linse, J. Chem. Phys. 113, 4359 (2000).

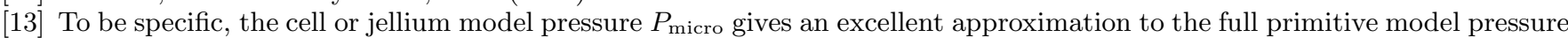
$P$ as e.g. computed in [12]. For a coupling $z^{3} Z_{c} \lambda_{B}^{2} / a^{2}>1$, non mean-field effects become important [4, 16].

[14] Y. Levin, E. Trizac and L. Bocquet, J. Phys.: Condens. Matt. 15, S3523 (2003).

[15] We emphasize that this agreement is systematically deteriorated or lost upon attempting to improve the original mean-field formulation, as proposed e.g. in S.N. Petris, D.Y.C. Chan and P. Linse, J. Chem. Phys. 118, 5248 (2003), or L.B. Bhuiyan and C.W. Outhwaite, J. Chem. Phys. 116, 2650 (2002). See [14] for a comparison.

[16] R.R. Netz, Eur. Phys. J. E 5, 557 (2001).

[17] B. Beresford-Smith, D.Y. Chan and D.J. Mitchell, J. Colloid Interface Sci. 105, 216 (1984).

[18] R. van Roij, J. Phys.: Condens. Matt. 12, A263 (2000).

[19] D. Antypov and C. Holm, Phys. Rev. Lett. 96, 088302 (2006).

[20] H.H. von Grünberg and L. Belloni, Phys. Rev. E, 62, 2493 (2000).

[21] S. Alexander, P.M. Chaikin, P. Grant, G.J. Morales, P. Pincus, and D. Hone, J. Chem. Phys. 80, 5776 (1984).

[22] L. Belloni, Colloid Surf. A 140, 227 (1998).

[23] E. Trizac L. Bocquet, M. Aubouy and H.H. von Grünberg, Langmuir 19, 4027 (2003).

[24] B. Jancovici, J. Stat. Phys. 28, 43 (1982).

[25] R.R. Netz, Phys. Rev. E 60, 3174 (1999).

[26] R. Bracewell, "The Fourier Transform and Its Applications", 3rd ed. New York: McGraw-Hill, 1999.

[27] L. Foret and A. Würger, Langmuir 20, 3842 (2004).

[28] J.G. Kirkwood and F.P. Buff, J. Chem. Phys., 19, 774 (1951).

[29] Within the jellium model in presence of an electrolyte, one has $\kappa^{4}=\kappa_{\text {res }}^{4}+\left(4 \pi \lambda_{B} \rho_{c} Z_{\text {eff }}\right)^{2}$ where $\kappa_{\text {res }}$ is the inverse Debye length in the reservoir against which the system is dialyzed. The precise form of the above relation is however not required for the present discussion. 